Text: 1,694 words (excluding summary, methods, references and legends)

Text: 4,133 (excluding references)

References: 35

4 main figures (approximate final size of figures: 10x13 cm)

9 supplementary figures

Expected number of Nature pages: 5

\title{
The tumour suppressor Pml regulates cell fate in the developing neocortex
}

\author{
Tarik Regad, Cristian Bellodi, Pierluigi Nicotera \& Paolo \\ Salomoni*
}

MRC Toxicology Unit, Leicester LE1 7JL, United Kingdom

*To whom the correspondence should be addressed. E-mail: ps90@le.ac.uk 
The control of cell fate in neural progenitor/stem cells is critical for nervous system development ${ }^{1,2}$. Nevertheless, the players involved are only partially known. We found that in the developing neocortex the expression of the tumour suppressor Pml is restricted to neural progenitor cells (NPCs). Remarkably, in Pml-/- cortices the overall number of proliferating NPCs is increased, while transition between the two major progenitor types, radial glial cells and basal progenitors is impaired. This in turn results in reduced differentiation and an overall decrease in thickness of the cortex wall. In NPCs, Pml regulates the subcellular distribution of the retinoblastoma protein $(\mathrm{pRb})$ and the protein phosphatase $1 \propto(\mathrm{PP} 1 \alpha)$, triggering pRb dephosphorylation. Together, these findings reveal an unexpected role of Pml in controlling the function of NPCs in the central nervous system.

The gene encoding the promyelocytic leukaemia protein (PML) is a tumour suppressor originally identified at the breakpoint of the $\mathrm{t}(15 ; 17)$ translocation found in acute promyelocytic leukaemia (APL) $\left(^{3,4}\right.$ and references cited therein). Pml is associated with a subnuclear structure known as the PML-nuclear domain (PML-ND), of which it is the essential component ${ }^{5-7}$. Pml is a potent growth suppressor in both cell lines and primary cells and regulates the tumour suppressors $\mathrm{p} 53$ and $\mathrm{pRb}{ }^{4,8-10}$. Recent studies suggest that Pml exerts its tumour suppressive function also outside the haemopoietic system ${ }^{4,11-13}$. In particular, Pml expression is lost in human tumours of multiple histological origins including tumours of the central nervous system, such as medulloblastoma ${ }^{4,11}$.

Correct regulation of cell cycle progression has been shown to be essential during the development of the nervous system (reviewed in ${ }^{1,2,14}$ ). In particular, the neural epithelium lying in the apical area of the developing neocortex gives rise to progenitors, post-mitotic neurons and macroglia in a temporally and spatially controlled fashion (reviewed in 1,2). How the balance between proliferation and differentiation of neural progenitors is maintained during early development and which are the main players involved remain central questions in the field of neurodevelopment ${ }^{14,15}$.

The role of Pml in the development of the nervous system is completely unexplored. We found that in the developing cerebral cortex Pml expression is 
confined to the neuroepithelial zone, where Nestin-positive neural stem / progenitor cells lie (Fig. 1a) ${ }^{16}$. By contrast, Pml was not detected in the cortical plate of Pml-/cortices and in early-born $\beta$ III Tubulin ( $\beta$ IIITub)-positive neurons in the ventricular zone (Fig. 1a and 1b). At the subcellular level, Pml accumulated in nuclear speckles (Fig. 1a). Pml expression was restricted to the progenitors zone also at postnatal day 0 (P0) and at P7 (Supplementary Fig. 1a, b). Cortical precursors divide to give rise to different types of differentiated cells that in turn migrate radially to specific layers in the cortical plate (reviewed in ${ }^{1}$ ). To test whether Pml plays a role in neocortex development, we analysed brains isolated from $P m l+/+, P m l+/-$ and $P m l-/-$ animals at P0 (total $n=32$ ), when neuronal differentiation is complete. Analysis of haematoxylin and eosin (H\&E)-stained coronal sections clearly showed that, while the overall organisation of the brain does not appear to be affected in Pml-/- mice, the cerebral cortex appears smaller (Fig. 1c). This effect was not solely restricted to the cortex, as also the size of the hippocampus appeared to be affected at P0 (Suppl. Fig. 2). Moreover, the width and length of cortex in whole brains was reduced (Fig. 1d) and the overall thickness of the cortex wall was also diminished (Fig. 1e). Finally, Pml-/adult brains show more modest albeit significant differences in thickness of the cortical wall, thus suggesting a degree of compensation at later stages (Suppl Fig. 3a, b). Taken together, these findings demonstrate that Pml loss affects the size of the cerebral cortex.

As mentioned above, the development of the neocortex relies on correct cell cycle regulation at the level of neural progenitor/stem cells in the $\mathrm{VZ}^{1,2}$. Therefore, as Pml is a growth suppressor, we reasoned that its loss could affect cell cycle in neural progenitors. We analysed the number of proliferating cells in the E15 VZ by using the Ki67 and PCNA proliferation markers and found that Pml-/- cortices presented an expanded pool of cycling cells (Fig. 2a and not shown). We also detected an increased number of mitotic cells at the apical side of the VZ using the mitotic marker phospho-histone H3 (Fig. 2b). In order to determine the number of cells in the S-phase of cell cycle, we injected 15-days pregnant females intraperitoneally (i.p.) with bromodeoxyuridine (BrdU). After a one-hour pulse, BrdU-labelled control cells were located basally in the $\mathrm{VZ}$ due to intrakinetic nuclear migration (Fig. 2c) ${ }^{1,2}$. The number of cells in $S$ phase was clearly elevated in the Pml-/- cortex, while nuclear positioning did not appear to be substantially affected 
(Fig. 2c). A similar increase in the number of Ki67-positive progenitors was observed in Pml-/- animals at P0 and P7 (Suppl. Fig. 4). Differences in proliferation of progenitors were also demonstrated in vitro using the neurosphere assay ${ }^{19}$. For instance, the diameter of neurospheres from Pml-/- E12 embryos was approximately twice the one of controls after 10 days in vitro (DIV10) at clonal density (Fig. 2d), and their number was also increased (not shown). Notably, Pml loss appeared to affect the transition between the two major types of progenitors present in the VZ, radial glial and basal progenitors ${ }^{17,18}$, as an increased number of Pax6-positive radial glial cells was accompanied by a reduction in basally-located progenitors, which were identified using the Tbr2 and P-H3 markers (Fig. 2e, f, g) 17, 18. Finally, developmental cell death was normal in mutant cortices at E15 (Suppl. Fig. 5), thus suggesting that the higher number of progenitors found in Pml-/- brains is caused by increased proliferation.

Two cell cycle parameters are believed to regulate the development of the neocortex: cell cycle exit and cell cycle re-entry ${ }^{14}$. We set out to investigate whether cell cycle exit is affected in Pml-deficient progenitors in vivo. To this end, we determined the number of BrdU+ and Ki67+ cells after a 24 hours BrdU pulse at E15 (n=3 Pml-/- and n=3 Pml+/- littermates). In this assay, BrdU+ / Ki67+ cells represent cells in cycle while, BrdU+ / Ki67- are cells that exited cell cycle. We found a twofold decrease in the number of cells exiting the cell cycle in Pml-/- cortices (Fig. 2h). Furthermore, the number of Ki67+/BrdU- cells present in the KO VZ was diminished, suggesting that a larger portion of proliferating cells enter S-phase compared to controls. This probably reflects shortening of the cell cycle. Taken together, these findings suggest that a greater proportion of $\mathrm{Pml-/}$ - progenitors re-enter cell cycle after mitosis and that Pml loss may alter the balance between cell cycle exit and reentry. Based on the models currently available ${ }^{14}$, decreased cell cycle exit would affect generation of neurons. Indeed, we revealed a significant decrease in the number of MAP2-positive neuronal cells at E15 (Fig. 3a, Suppl. Fig. 6a) and P0 (Fig. 3b, Supplementary Fig. 6b) in the Pml-/- cortical plate. However, the layered structure of the cortex and neuronal specification did not appear to be affected in Pml-/- animals, as deep layer 6 (Foxp2+) and layers 3-4-5 (Foxp1+), despite containing a reduced number of cells, were correctly positioned (Suppl. Fig. 7). Generation of astrocytes (GFAP+) and oligodendrocytes (MBP+) was also affected in the Pml-/- cerebral 
cortex at P7 (Suppl. Fig. 6c). In vitro differentiation assays demonstrated that Pml loss results in an overall reduction of MAP2+ neurons, accompanied by an increase in Nestin+ cells (DIV5; Fig. 3c, Suppl. Fig. 8). Also differentiation into astrocytes and oligodendrocytes was affected in Pml-/- cultures (DIV10; Fig. 3c, Suppl. Fig. 8). Reintroduction of PML I in Pml-/- progenitors results in decreased size of neurospheres and rescued differentiation (Fig. 3d, e). Interestingly, mutation of the PML SUMOylation sites governing PML-ND formation ( $\triangle$ SUMO-PML I) ${ }^{7}$ abolished the effect on proliferation and differentiation in Pml-deficient cells (Fig. 3d, e), thus suggesting that formation of PML-ND is a prerequisite for PML I activity in cortical progenitors.

Among Pml interactors, the Retinoblastoma protein (pRb) ${ }^{9,}{ }^{20-22}$ has an established role in nervous system development and pathogenesis of brain tumours ${ }^{23-}$ 28 . However, $\mathrm{pRb}$ function at the level of neural precursors is still unclear. We found that in cortical progenitors $\mathrm{pRb}$ accumulates in nuclear speckles, which partially colocalize with PML-NDs (Fig. 4a). In contrast, in Pml-/- cells pRb was more nucleoplasmic and also accumulated in the cytoplasm (Fig. 4a). Similar staining pattern was obtained using a different anti-pRb antibody (Suppl. Fig. 9a). These changes did not occur in the cortical plate, where Pml is not normally expressed, thus confirming the specificity of the observed alterations (Fig. 4a; Suppl. Fig. 9a, c). Furthermore, we found that $\mathrm{pRb}$ and Pml interact in neural progenitors (Fig. 4b). pRb is inactivated through phosphorylation by cyclin-dependent kinases ${ }^{29}$. Interestingly, in Pml-/- cortical progenitors cells pRb was hyperphosphorylated, thus suggesting that Pml regulates pRb phosphorylation status (Fig. 4c), as proposed by previous studies 9 , 22. Cyclin-dependent phosphorylation of $\mathrm{pRb}$ is counteracted by the activity of the protein phosphatase $1 \alpha(\mathrm{PP} 1 \alpha)^{30,31}$, which has been recently implicated in neocortex development ${ }^{32}$. We found that Pml, $\mathrm{pRb}$ and PP1 $\alpha$ colocalize in the nucleus of neuronal progenitors both in vivo and in vitro (Fig. 4d, e; Suppl. Fig. 9b, c). Instead, in Pml-/- cortices and isolated cortical progenitors PP1 $\alpha$ localization changed to a more diffuse nuclear staining and cytoplasmic accumulation (Fig. 4d, e and Suppl. Fig. 9b, c). Finally, both $\mathrm{pRb}$ and PP1 $\alpha$ are found in anti-Pml immunoprecipitates, thus suggesting that the three proteins form a nuclear complex (Fig. 4f). Reintroduction of PML I isoform in Pml-/- progenitors resulted in decreased $\mathrm{pRb}$ phosphorylation (Fig. $4 \mathrm{~g}$ ). In contrast, the $\triangle$ SUMO-PML I mutant failed to affect $\mathrm{pRb}$ 
phosphorylation (Fig. 4g). Finally, the effect on phosphorylation was completely abolished in the presence of the PP1 $\alpha$ inhibitor tautomycetin (not shown).

Overall, our results provide evidence of a novel Pml role in regulating cell fate in neural progenitor/stem cells. In particular, Pml loss appears to affect proliferation of radial glial cells and to inhibit the transition to basal progenitors. This in turn leads to impaired differentiation and reduced size of the cortex. As for the underlying mechanism, we propose a model by which Pml controls cell cycle in neural progenitor/stem cells by affecting $\mathrm{pRb}$ phosporylation status, thus potentially revealing a novel role of $\mathrm{pRb}$ outside the pool of maturing neurons $24,25,28,33$. More generally, our work shows that an increased number of progenitors in the developing cerebral cortex does not necessarily result in augmented neurogenesis, but instead it can lead to a block of differentiation and in turn to a decrease in cortex size. Finally, our findings, together with the reported loss of PML expression in human brain tumours ${ }^{11}$, opens up the possibility that alterations of PML function may be involved in the onset of neoplastic disorders originating from the neuroepithelial compartment of the nervous system. 


\title{
METHODS
}

\author{
Animals \\ Pml-/- animals were a generous gift from Dr. Pier Paolo Pandolfi (Harvard \\ University, Boston, USA). Animals were backcrossed to the sv129 S2 strain for eight \\ generations. Animals were bred and subjected to listed procedures under the Project \\ Licence no. 80-2085, released from the Home Office.
}

\section{Antibodies}

Anti-PML (05-718; 1/500 for IB, 1/100 for IHC, IF and IP) from Upstate/Millipore; Anti-BrdU-fluorescin from Roche (1202693; 1/1000), anti-MAP2 (ab11267; 1/50), anti-FOXP1 (ab16645; 1/200), anti-FOXP2 (ab16046; 1/2500) and anti-Rb (ab6075; $1 / 50$ for IHC and IF) from Abcam; anti-MBP (AB980; 1/500), anti-PML (AB1370) and anti-Nestin (MAB353; 1/500) from Chemicon; anti-pRb (554136; 1/100 for IB) from BD Pharmingen; anti- $\beta$-actin from Sigma (A2172; 1/5000); anti GFAP (Z0334; 1/500) from Dako, anti-PP1 $\alpha$ (sc-6104; $1 / 100$ for IHC and IF, $1 / 500$ for IB) from Santa Cruz Biotechnology; anti-Ki67 (NCL-Ki67p; 1/500) from Novocastra; antiPax6 (supernatant concentrate; 1/200) from DSHB/University of Iowa; anti-Tbr2 (IHC/IF 1:2,000; a kind gift from Dr Hevner, Seattle Children's Hospital Research Institute, Seattle, USA) anti-active caspase 3 (9661S; 1/200) from Cell signalling; anti-phospho H3 (1:200) from Upstate/Millipore.

\section{Immunohistochemistry and immunofluorescence}

E12, P0 or P7 brains were fixed in 4\% paraformaldehyde and embedded in paraffin. The sections were de-waxed, re-hydrated in graded alcohols, rinsed in distilled water and antigen retrieval treated for 30 minutes $(0.01 \mathrm{M}$ citric acid at $\mathrm{pH} 6.0)$ in pressure cooker at 700 Watts. For staining of isolated cells, cortical cells were prepared and treated as explained below and fixed in $4 \%$ paraformaldehyde. The sections or cortical cells were washed in PBS 1x for 3X10 minutes, blocked in $10 \%$ goat serum in $0.1 \%$ PBS-Tween followed by overnight first antibody incubation (in blocking solution), washed three times 10 minutes with PBS $1 \mathrm{x}$, an $1 \mathrm{~h}$ incubation with secondary antibody (in blocking solution) then three times wash with PBS $1 \mathrm{x}$ and counterstained with DAPI and mounted for confocal microscopy. For triple staining experiments, all immunofluorescence steps were performed using 5\% bovine serum albumin (BSA) as blocking reagent. Two different images of each section or cortical cells were taken and the number of cells labelled was counted ( $n=3$ PML null compared to $n=3$ control littermates).

\section{BrdU labelling}

Pregnant females at E15 days of gestation were injected intraperitoneally for 1hour with $50 \mu \mathrm{g} / \mathrm{g}$ body mass bromodeoxyuridine (BrdU). One hour after, the females were culled and the embryos removed, fixed in $4 \%$ paraformaldehyde and embedded in paraffin. Sections were stained with anti-BrdU-fluorescin isothiocyanate (FITC) antibody. The number of BrdU-labeled cells was determined by counting BrdU+ cells from two confocal microscopy images of corresponding sections from 3 independent experiments ( $n=3$ PML null compared to $n=3$ control littermates).

For the cell cycle exit experiment, pregnant females at E14 were injected with BrdU as described above for 24 hours, culled and the embryos removed, fixed in $4 \%$ parafolmaldehyde and embedded in paraffin for sectioning. The sections were co- 
stained with anti-BrdU-fluorescin isothiocyanate and Ki67 antibodies. The cells counted in this experiment are the ones that exited the cell cycle (BrdU + only) and are localised in the Intermediate zone after radial migration. The cells that are still proliferating or remain in the cell cycle are BrdU+ and Ki67+ and are localised in the VZ.

\section{Immunoblotting and Immnunoprecipitation}

For immunoblotting, cortical neurospheres were collected, washed with 1x PBS then lysed in $1 \mathrm{x}$ solution containing $50 \mathrm{mM}$ Tris $-\mathrm{HCl}(\mathrm{pH} 6.8), 100 \mathrm{mM}$ dithiothreitol, $2 \%$ SDS, 0.1 bromophenol blue and 10\% glycerol prior loading on Tris/glycine SDSpolyacrylamide gels. For immunoprecipitation, the cortical cells were lysed in immunoprecipitation (IP) buffer $(0.05 \mathrm{M}$ Tris $\mathrm{pH} 7.4,0.15 \mathrm{M} \mathrm{NaCl}, 0.5 \%$ Triton, $0.001 \mathrm{M}$ EDTA). The extracts were precleared for 1 hour at $4^{\circ} \mathrm{C}$ using Protein G PlusAgarose beads (IP08, Calbiochem) and anti-IgG2b (Dako). For the antibody binding, anti-PML was incubated with agarose beads for 2 hours in IP buffer prior adding the precleared extracts overnight for immunoprecipitation.

\section{Cortical cell culture}

Cortical cultures were prepared using reagents from Stem Cell Technologies and following the manufacturer's protocol and previously reported protocols ${ }^{19}$. For the neurospheres culture, the cortical cells were plated at clonal density $\left(20 \times 10^{3}\right.$ cells $/ \mathrm{ml}$ in 24-well plates) in complete neurocult neural stem cells (NSC) proliferation medium containing $10 \mathrm{ng} / \mathrm{ml}$ bFGF (Peprotech) and cultured for 10 days in vitro (DIV). For the differentiation assay, single cell suspensions of cortical cells were plated at $5 \times 10^{5}$ cells/well on poly-L-ornithine glass coverslips in complete neurocult NSC differentiation media. At DIV5 cells were fixed in $4 \%$ paraformaldehyde and analyzed for expression of the progenitor marker Nestin and the neuronal marker MAP2 by immunofluorescence. Instead, at DIV10 cells were stained with antibodies against Nestin and MBP and GFAP, which are markers for oligodendrocytes and astrocytes, respectively ${ }^{34}$. The PP1 $\alpha$ inhibitor (InSolution Tautomycetin, $S$. greseochrogenes; cat no. 580550 Calbiochem) was added to the neurospheres culture $(5 \mu \mathrm{M})$ for 5.5 hours.

\section{Retroviral expression of PML}

PML isoform V cDNA (kind gift from David Grimwade) was cloned into the EcoRI site of pBabePuro (pBabe). Then, a PCR-amplified fragment containing the PML Ispecific C-term was cloned into Mlu I/ Sal I sites of PML V pBabe to obtain PML I pBabe. The PML I mutant lacking PML's three SUMOylation sites ( $\triangle$ SUMO PML I pBabe) was generated by site-directed mutagenesis. Cortical cells were infected with high-titer pBabe, pBabe-PML I or pBabe $\triangle$ SUMO PML I retroviral supernatants and selected in puromycin (Sigma) for 48 hours ${ }^{35}$. 


\section{ACKNOWLEDGMENTS}

A special thank to Dr Hevner (Seattle Children's Hospital Research Institute, Seattle, USA) for the anti-Tbr2 antibody, and to David Dinsdale, Jenny Edwards and Richard Edwards (MRC Toxicology Unit, Leicester, UK) for support and assistance with immunohistochemistry. We also thank Christine Watson (University of Cambridge, UK), David Read, Gerry Melino, Richard Knight, and Melania Capasso (MRC Toxicology Unit, Leicester, UK) for critical discussion. P.S., P.N., T.R., and C.B. are supported by the MRC, UK. C.B. is a PhD student at the University of Leicester.

\section{AUTHOR CONTRIBUTION}

T.R. performed most of the experiments and contributed to the writing of the manuscript; C.B. performed a number of experiments; P.N. provided expertise; P.S. supervised the research project, performed a number of experiments and wrote the manuscript.

\section{REFERENCES}

1. Campbell, K. Cortical neuron specification: it has its time and place. Neuron 46, 373-6 (2005).

2. Guillemot, F. Cellular and molecular control of neurogenesis in the mammalian telencephalon. Curr Opin Cell Biol 17, 639-47 (2005).

3. Melnick, A. \& Licht, J. D. Deconstructing a disease: RARalpha, its fusion partners, and their roles in the pathogenesis of acute promyelocytic leukemia. Blood 93, 3167-215 (1999).

4. Salomoni, P., Ferguson, B. J., Wyllie, A. H. \& Rich, T. New insights into the role of PML in tumour suppression. Cell Res 18, 622-40 (2008).

5. Ishov, A. M. et al. PML is critical for ND10 formation and recruits the PMLinteracting protein daxx to this nuclear structure when modified by SUMO-1. J Cell Biol 147, 221-34 (1999).

6. Jensen, K., Shiels, C. \& Freemont, P. S. PML protein isoforms and the RBCC/TRIM motif. Oncogene 20, 7223-33 (2001).

7. Bernardi, R. \& Pandolfi, P. P. Structure, dynamics and functions of promyelocytic leukaemia nuclear bodies. Nat Rev Mol Cell Biol 8, 1006-16 (2007).

8. Bischof, O. et al. Deconstructing PML-induced premature senescence. Embo J 21, 3358-69 (2002).

9. Ferbeyre, G. et al. PML is induced by oncogenic ras and promotes premature senescence. Genes Dev 14, 2015-27 (2000).

10. Pearson, M. et al. PML regulates p53 acetylation and premature senescence induced by oncogenic Ras. Nature 406, 207-10 (2000).

11. Gurrieri, C. et al. Loss of the tumor suppressor PML in human cancers of multiple histologic origins. J Natl Cancer Inst 96, 269-79 (2004).

12. Scaglioni, P. P. et al. A CK2-dependent mechanism for degradation of the PML tumor suppressor. Cell 126, 269-83 (2006). 
13. Trotman, L. C. et al. Identification of a tumour suppressor network opposing nuclear Akt function. Nature 441, 523-7 (2006).

14. Dehay, C. \& Kennedy, H. Cell-cycle control and cortical development. Nat Rev Neurosci 8, 438-50 (2007).

15. Caviness, V. S., Jr. et al. Cell output, cell cycle duration and neuronal specification: a model of integrated mechanisms of the neocortical proliferative process. Cereb Cortex 13, 592-8 (2003).

16. Frederiksen, K. \& McKay, R. D. Proliferation and differentiation of rat neuroepithelial precursor cells in vivo. J Neurosci 8, 1144-51 (1988).

17. Haubensak, W., Attardo, A., Denk, W. \& Huttner, W. B. Neurons arise in the basal neuroepithelium of the early mammalian telencephalon: a major site of neurogenesis. Proc Natl Acad Sci U S A 101, 3196-201 (2004).

18. Hevner, R. F. From radial glia to pyramidal-projection neuron: transcription factor cascades in cerebral cortex development. Mol Neurobiol 33, 33-50 (2006).

19. Tropepe, V. et al. Distinct neural stem cells proliferate in response to EGF and FGF in the developing mouse telencephalon. Dev Biol 208, 166-88 (1999).

20. Alcalay, M. et al. The promyelocytic leukemia gene product (PML) forms stable complexes with the retinoblastoma protein. Mol Cell Biol 18, 1084-93 (1998).

21. Labbaye, C. et al. Expression and role of PML gene in normal adult hematopoiesis: functional interaction between PML and $\mathrm{Rb}$ proteins in erythropoiesis. Oncogene 18, 3529-40 (1999).

22. Mallette, F. A., Goumard, S., Gaumont-Leclerc, M. F., Moiseeva, O. \& Ferbeyre, G. Human fibroblasts require the $\mathrm{Rb}$ family of tumor suppressors, but not p53, for PML-induced senescence. Oncogene 23, 91-9 (2004).

23. Jacks, T. et al. Effects of an Rb mutation in the mouse. Nature 359, 295-300 (1992).

24. Lipinski, M. et al. Comparison of exercise test scores and physician estimation in determining disease probability. Arch Intern Med 161, 2239-44 (2001).

25. Ferguson, K. L. et al. Telencephalon-specific Rb knockouts reveal enhanced neurogenesis, survival and abnormal cortical development. Embo J 21, 333746 (2002).

26. Marino, S., Hoogervoorst, D., Brandner, S. \& Berns, A. Rb and p107 are required for normal cerebellar development and granule cell survival but not for Purkinje cell persistence. Development 130, 3359-68 (2003).

27. MacPherson, $\mathrm{D}$. et al. Cell type-specific effects of $\mathrm{Rb}$ deletion in the murine retina. Genes Dev 18, 1681-94 (2004).

28. Ferguson, K. L. et al. A cell-autonomous requirement for the cell cycle regulatory protein, Rb, in neuronal migration. Embo J 24, 4381-91 (2005).

29. Sherr, C. J. Cancer cell cycles. Science 274, 1672-7 (1996).

30. Alberts, A. S., Thorburn, A. M., Shenolikar, S., Mumby, M. C. \& Feramisco, J. R. Regulation of cell cycle progression and nuclear affinity of the retinoblastoma protein by protein phosphatases. Proc Natl Acad Sci U S A 90, 388-92 (1993).

31. Durfee, T. et al. The retinoblastoma protein associates with the protein phosphatase type 1 catalytic subunit. Genes Dev 7, 555-69 (1993).

32. Kim, T. H., Goodman, J., Anderson, K. V. \& Niswander, L. Phactr4 regulates neural tube and optic fissure closure by controlling PP1-, Rb-, and E2F1regulated cell-cycle progression. Dev Cell 13, 87-102 (2007). 
33. de Bruin, A. et al. $\mathrm{Rb}$ function in extraembryonic lineages suppresses apoptosis in the CNS of Rb-deficient mice. Proc Natl Acad Sci U S A 100, 6546-51 (2003).

34. Shen, Q. et al. The timing of cortical neurogenesis is encoded within lineages of individual progenitor cells. Nat Neurosci 9, 743-51 (2006).

35. Bellodi, C. et al. Cytoplasmic function of mutant promyelocytic leukemia (PML) and PML-retinoic acid receptor-alpha. J Biol Chem 281, 14465-73 (2006). 


\section{FIGURES LEGENDS}

Figure $1 \mathrm{Pml}$ expression is confined to neural progenitor cells

(a) Expression of Pml in coronal section of Pml+/- E15 embryo neocortex. Sections were also stained for Nestin, which is a marker of progenitors in the ventricular zone (VZ), and Map2, which is a marker of mature neurons in the cortical plate (CP). Pml expression encompasses the Nestin progenitor domain but is absent in the Map2+ domain. Close-up images show Pml accumulation in nuclei of neural progenitor cells (NPC, top right panel). Bottom left panel shows anti-Pml staining in the Pml-/cortex. Scale bars: $200 \mu \mathrm{m}$ and $10 \mu \mathrm{m}$. (b) $\beta$ IIITubulin ( $\beta$ IIITub)+ cells are negative for Pml expression in the VZ and CP. Coronal section of Pml+/- E15 embryo cerebral cortex were stained with anti- $\beta$ IIITub and anti-Pml antibodies. Arrowheads indicate $\beta$ IIITub+ cells in the VZ. (c) Expression of Pml in coronal section of Pml+/- cerebral cortex at P0 was performed as in Fig. 1. (d) Expression of Pml at the level of the lateral ventricles at P7. Scale bars: $200 \mu \mathrm{m}$. For both c and d, haematoxylin and eosin (H\&E)-stained coronal sections stained shown to highlight areas on which the immunohistochemical analysis was performed (boxed).

Figure $2 \mathrm{Pml}$ regulates the size of the cerebral cortex.

(a) Images of Pml-/- brains ( $\mathrm{n}=12$ mice) show a clear reduction in size when compared to $P m l+/+(\mathrm{n}=5$ mice $)$ and $P m l+/-(\mathrm{n}=15$ mice $)$. Graphs show width and length of cortices from P0 Pml-/-, Pml +/+ and Pml +/- animals. Graphs show mean \pm s.e.m. of cortex width and length $(\mathrm{p}<0.0001$, unpaired t-test, two tails; also for the following figures, unless stated otherwise). (b) Coronal sections stained with haematoxylin and eosin $(\mathrm{H} \& \mathrm{E})$ indicate smaller brain size in the $\mathrm{Pml}-/$ - compared to $\mathrm{Pml}+/$ - animals at P0. (c) Thickness of the cortex wall is reduced in Pml-deficient P0 brains. Coronal sections of Pml+/- and Pml-/- P0 brains were stained with H\&E and the cortex thickness was measured (Axiovert, Germany). Histogram shows mean \pm s.e.m. of cortex thickness $(* * \mathrm{p}=0.00025)$. (d) Coronal sections through $P m l+/-$ and Pml-/- adult brains were stained with $\mathrm{H} \& \mathrm{E}$ and analysed using Axiovert for cortex wall thickness. A to E indicate areas $(500 \mu \mathrm{m})$ of brain sectioning, while numbers indicate sections $(10 \mu \mathrm{m})$ for each area. Hippocampus is present in sections starting at D2. Graph shows means \pm s.e.m $(n=3$ each genotype; B3 * $\mathrm{p}=0.0273$; $\mathrm{C} 1 * \mathrm{p}=0.0384$; C2 $* \mathrm{p}=0.0154$; C3 $* \mathrm{p}=0.0296$; D1 $* \mathrm{p}=0.010$; D2 *p=0.0493). b, Representative images of section B3. Scale bar: $200 \mu \mathrm{m}$. Striatum is indicated (S).

\section{Figure 3: Pml controls proliferation and the cell cycle exit in neuronal progenitors.}

(a) Increased number of Ki67+ cells in Pml-/- E15 embryos. Immunohistochemistry with anti-Ki67 proliferation marker on coronal sections through the cerebral cortex of E15 Pml+/- and Pml-/- embryos. (b) Increased number of mitotic cells at the apical surface of Pml-/- E15 embryos. Immunohistochemistry with anti-phosphorylated histone $\mathrm{H} 3(\mathrm{P}-\mathrm{H} 3)$ and $\beta$ IIITub antibodies on coronal sections through the cerebral cortex of E15 Pml+/- and Pml-/- embryos. Histogram shows mean \pm s.e.m. of number of $\mathrm{P}-\mathrm{H} 3+$ cells $(* * \mathrm{p}=0.0077)$. (c) Increased number of cells in S-phase in Pmldeficient ventricular zone (VZ). Anti-BrdU-fluorescin isothiocyanate (FITC; green) on coronal sections through the cerebral cortex of E15 Pml+/- and Pml-/- embryos isolated from pregnant mice after a one hour BrdU pulse. Histogram shows mean \pm stdev of BrdU+ cells in images of 3 different stained sections from 3 different embryos for each genotype ( $\mathrm{n}=3 \mathrm{Pml} /$ - compared to $\mathrm{n}=3 \mathrm{Pml}+/$ - littermates control; 
$* p=0.0317)$. (d) Immunohistochemistry with anti-BrdU-FITC and anti-Ki67 on coronal sections through the cerebral cortex of E15 Pml+/- and Pml-/- embryos obtained from pregnant mice injected with BrdU for 24 hours. The number of BrdU+/Ki67- (cells that exited cell cycle) and BrdU+/Ki67+ (cells in cycle) present in the Pml-/- VZ and cortical plate was analyzed. The graph expresses the mean \pm stdev of the number of BrdU+/Ki67+ in Pml-/- versus Pml+/- intermediate zone in two images of two different stained sections from two different embryos for each genotype ( $\mathrm{n}=2 \mathrm{Pml}-/$ - compared to $\mathrm{n}=2 \mathrm{Pml}+/$ - littermates controls; * $\mathrm{p}=0.0196)$. Scale bar: $20 \mu \mathrm{m}$ in.

Figure $4 \mathrm{Pml}$ loss skews the composition of neural progenitors subtypes in the developing neocortex, (a) Increased number of radial glial cells in Pml-deficient cortices. Immunohistochemistry with anti-Pax6 and $\beta$ IIITub antibodies through the cerebral cortex of E15 Pml+/- (top) and Pml-/- (bottom) embryos. (b) Reduction in basal progenitors in Pml-/- cortices. Brains were stained with an antibody against Tbr2, a marker of basal progenitors. (c) reduction basally located mitoses $(\mathrm{P}-\mathrm{H} 3+)$ in Pml-deficient brains. Histogram shows mean \pm s.e.m of $\mathrm{P}-\mathrm{H} 3+$ cells numbers (11 separate sections each genotype; $* \mathrm{p}=0.0178$ ).

Figure 5 The overall number of neurons is reduced in Pml-/- cortices, while cortical architecture is not affected

(a) Immunohistochemistry with the neuronal marker Map2 on E15 Pml+/- and Pml-/coronal sections through the cerebral cortex shows a reduced size of the cortical plate in Pml-/- embryos compared to Pml+/- (Left panel). Less Map2+ (neurons) were generated in the Pml-/- cerebral cortex compared to the Pml+/- (right panel). Scale bar: $200 \mu \mathrm{m}$. (b) Immunohistochemistry with the neuronal marker Map2 on P0 Pml+/and Pml-/- coronal sections through the cerebral cortex shows a reduced size of the cortical plate in Pml-/- embryos compared to the Pml+/- (Left panel). Scale bar: $200 \mu \mathrm{m}$. The number of MAP2+ cells was determined by counting Dapi+/MAP2+ cells in two images of two different sections for each genotype ( $\mathrm{n}=2 \mathrm{Pml} / \mathrm{-}$ - and $\mathrm{n}=2$ Pml +/- littermates; a **p $=0.002, \mathrm{~b} * * \mathrm{p}=0.0028)$. $(\mathbf{c}, \mathbf{d})$ The overall architecture of the cortex is maintained in Pml-deficient brains. Immunohistochemistry with antiFoxp1 (c) and anti-Foxp2 (d) on coronal sections through the cerebral cortex from Pml+/- (upper panels) and Pml-/- (lower panels) pups at P0. While Foxp1+ neurons are found in layers 3,4,5, Foxp2 specifies layer neurons. The graphs show the number of Foxp1+ and Foxp2+ cells from two similarly sized images from 2 different stained sections for each genotype ( $\mathrm{n}=2 \mathrm{Pml} / /$ - compared to $\mathrm{n}=2 \mathrm{Pml}+/$ - littermates control). Scale bars: $200 \mu \mathrm{m}(\mathrm{c})$ and $100 \mu \mathrm{m}(\mathrm{d}){ }^{*} \mathrm{p}=0.0162$ (c), ${ }^{*} \mathrm{p}=0.0294$ (d).

\section{Figure 6 Pml-deficient NPCs display increased proliferation and impaired differentiation in vitro}

(a) Pml loss causes increased proliferation in neurospheres cultures derived from E12 NPCs. Representative image showing the size of Pml-/- primary neurospheres compared to Pml+/-. Histogram shows mean \pm stdev of neurosphere diameter, which measured in 10 live images / genotype $(\mathrm{n}=2 \mathrm{Pml}+/$ - and $\mathrm{n}=2 \mathrm{Pml}+/$ - littermates; ** $\mathrm{p}=0.0056$ ). (b) Differentiation is impaired in Pml-/- cultures. Neurosphere differentiation assays using cortical progenitors from $\mathrm{Pml}+/$ - (upper set of panels) and Pml-/- embryos at E12 (lower set of panels). The number of Nestin+ (precursors), Map2+ (neurons), GFAP+ (astrocytes) and MBP+ (oligodendrocytes) were counted. The percentage of Nestin versus differentiated cells (Map2+, GFAP+ or MBP+) was 
determined in two immunoflurescence images for each embryo ( $\mathrm{n}=3 \mathrm{Pml} / \mathrm{-}$ - and $\mathrm{n}=3$ Pml +/- littermate embryos; left panel: $* * \mathrm{p}=0.0018$ and $* \mathrm{p}=0.0351$; middle panel: $* * \mathrm{p}=0.0015$ and $* * \mathrm{p}=0.0085$; right panel: $* \mathrm{p}=0.0256$ and ${ }^{*} \mathrm{p}=0.0015$. (d, e) Reintroduction of PML I, but not of PMLI $\triangle$ SUMO reduces proliferation and rescues differentiation in vitro. NPCs were infected with PML I or PMLI $\triangle$ SUMO pBabe viruses and assayed for their proliferation and differentiation capacity. (d) Neurosphere proliferation assays were performed as described above. The diameter of neurospheres was determined in 16 live images/sample in culture of uninfected or infected cells; representative of two independent experiments. (e) PML I, but not PMLI $\triangle$ SUMO promotes differentiation of progenitors in vitro. Neurosphere differentiation assay were performed as in (a). Representative of two independent experiments.

\section{Figure $7 \mathrm{Pml}$ interacts and colocalises with $\mathbf{p R b}$}

(a) Immunohistochemistry with anti-Pml and anti-pRb antibodies on coronal sections from E15 Pml+/- (first and third set of panels from top) and Pml-/- (second and fourth set of panels) embryos (40X; scale bar: $50 \mu \mathrm{m}$ ). High magnification images of the VZ (third and fourth set of panels) show $\mathrm{pRb}$ and Pml colocalization (63X). The last set of panels shows that $\mathrm{pRb}$ accumulates in both the nucleus and the cytoplasm in the Pml-/- VZ. Arrowhead indicates cells in the Pml-/- cortical plate displaying nuclear $\mathrm{pRb}$ staining. Scale bars: $5 \mu \mathrm{m}$ (lower two set of panels). (b) $\mathrm{pRb}$ is found in immunoprecipitates from $\mathrm{Pml}+/$ - but not $\mathrm{Pml} / /$ - extracts. Immunoprecipitation with an anti-Pml antibody from $\mathrm{Pml}+/-$ and $\mathrm{Pml}-/$ - extracts. Input lanes show $\mathrm{pRb}$ and $\mathrm{Pml}$ expression in $\mathrm{Pml}+/$ - and Pml-/- NPCs. $\beta$-actin was measured as loading control.

\section{Figure 8 Pml-mediated control of PP1 $\alpha$-dependent dephosphorylation of $\mathrm{pRb}$ in the NPCs}

(a) $\mathrm{pRb}$ is hyperphosphorylated in Pml-deficient NPCs. Immunoblotting of extracts from $\mathrm{Pml}+/-$ and $\mathrm{Pml} / /$ - NPCs using an anti-pRb antibody shows an increase in the level of the pRb hyper-phosphorylated form (*) in Pml-/- extracts. The expression of Pml was analysed using an anti-Pml antibody. Anti- $\beta$-actin antibody was used as loading control. (b) PP1 $\alpha$ localisation is altered in Pml-/- cortices. Immunohistochemistry with anti-Pml (red), anti-pRb (green) and anti-PP1 $\alpha$ (purple) antibodies was performed on coronal sections from Pml+/- E15 embryos (ventricular zone). (c) PML partially colocalises with $\mathrm{pRb}$ and PP1a in vivo, and PP1 $\alpha$ localisation is altered in Pml-deficient cortices. Sections were stained as in (b) (scale bars: $5 \mu \mathrm{m}$ ). (d) Immunofluorescence of control (+/-) and Pml-/- cortical NPCs stained with anti-Pml (red), -pRb (green) and -PP1 $\alpha$ (purple) antibodies. DAPI was used for nuclear staining. Scale bars: $5 \mu \mathrm{m}$. (e) Both PP1 $\alpha$ and $\mathrm{pRb}$ are found in antiPml immunoprecipitates. Immunoprecipitation of Pml was performed as in Fig. 7c using extracts from $\mathrm{Pml}+/$ - and $\mathrm{Pml} / /$ - NPCs. Immunoprecipitates were run on a $10 \%$ SDS-PAGE and probed with an anti-pRb, Pml and PP1 $\alpha$ antibody (upper panel). Inputs show pRb, Pml and PP1 $\alpha$ expression. (f) PML I, but not PMLI $\triangle$ SUMO, expression in Pml-deficient NPCs reduces pRb phosphoryation. Hyper- and hypophosphorylated forms of $\mathrm{pRb}$ were analyzed using an anti-pRb antibody in extracts from Pml-/- progenitors uninfected or infected with pBabe pBabe-PMLI and pBabePMLI $\triangle$ SUMO viruses. Pml and PP1 $\alpha$ were also detected using specific antibodies. $\beta$-actin was measured as loading control. 


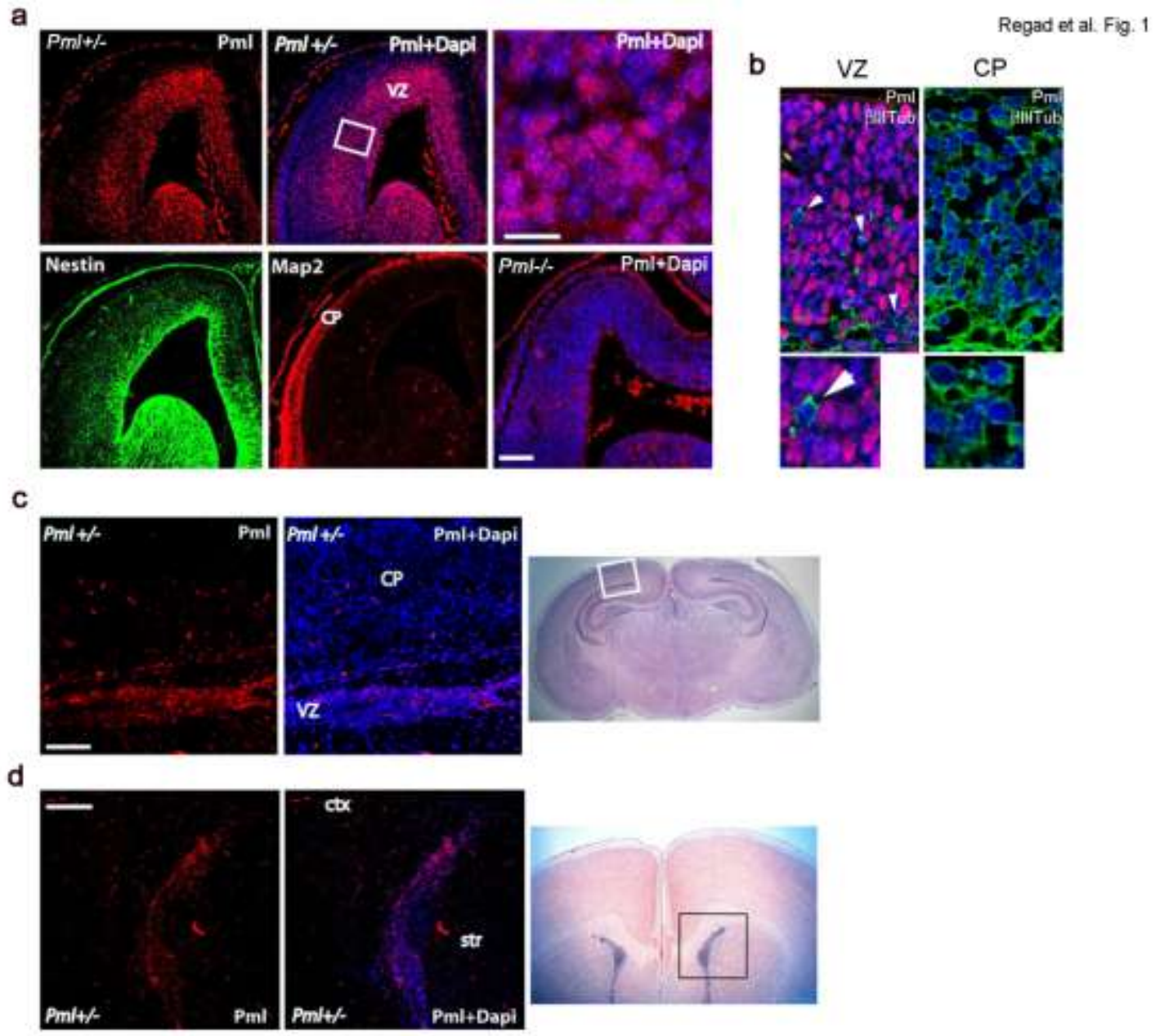


a

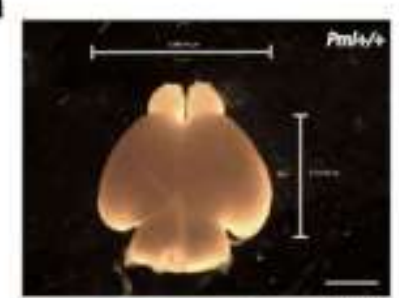

$\longmapsto$ Pulte

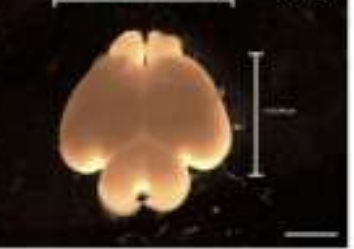

$\longmapsto \quad$ Ams

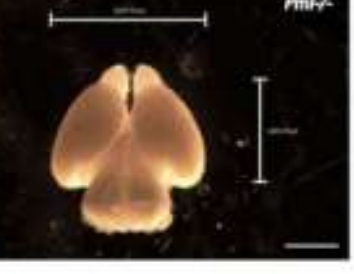

c

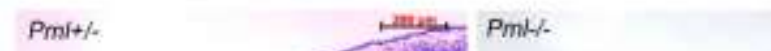

d

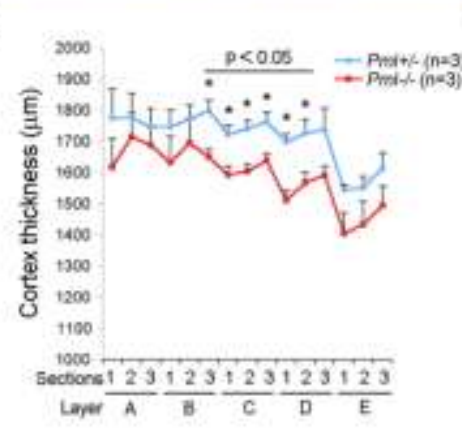

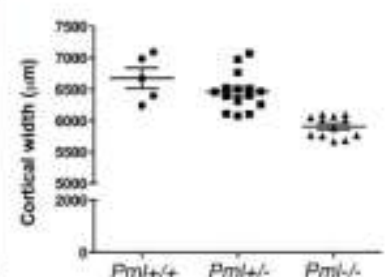

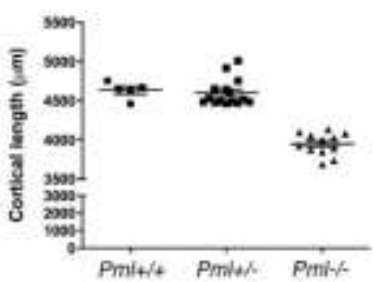

b

Regad et al. Fig. 2

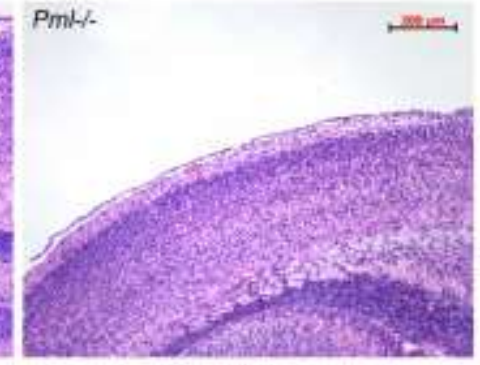

e

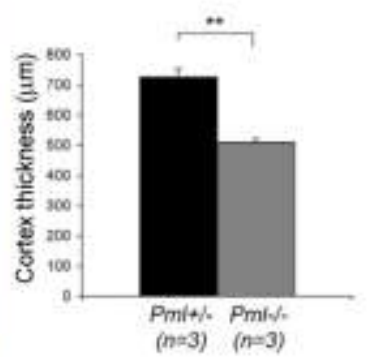

s

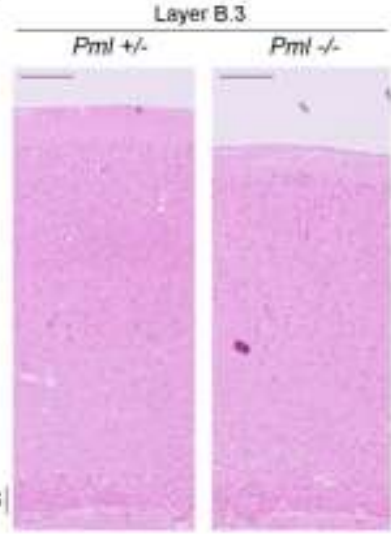




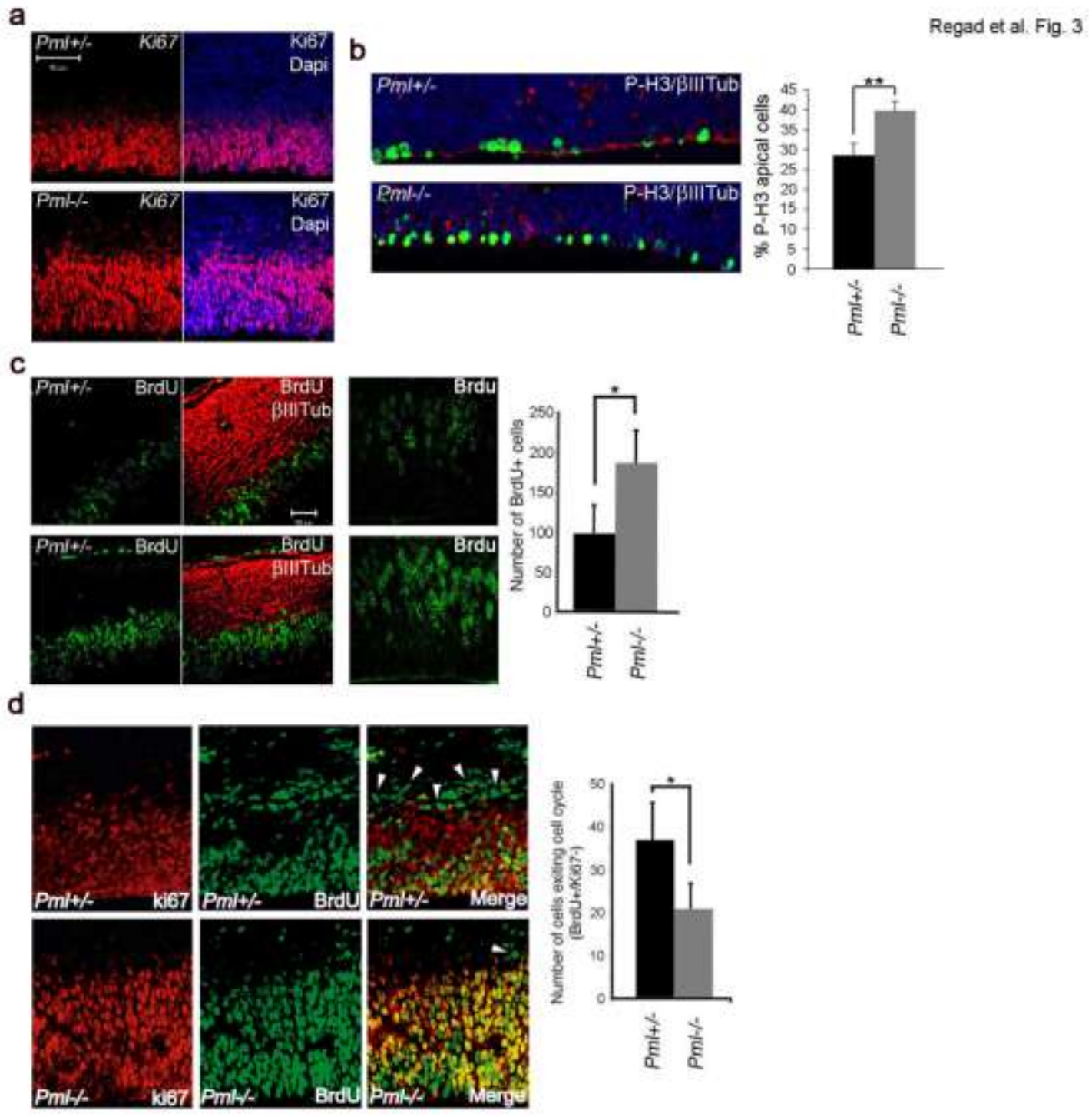




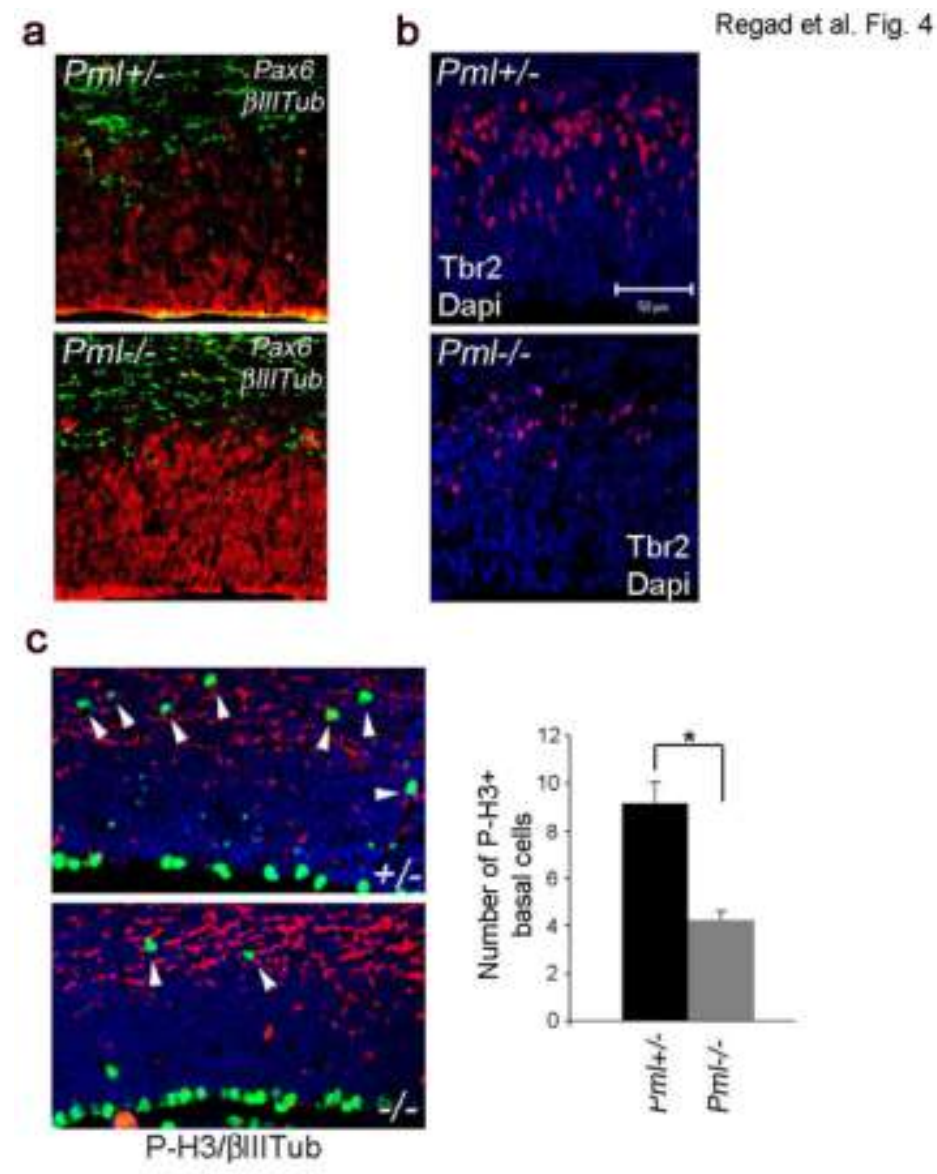




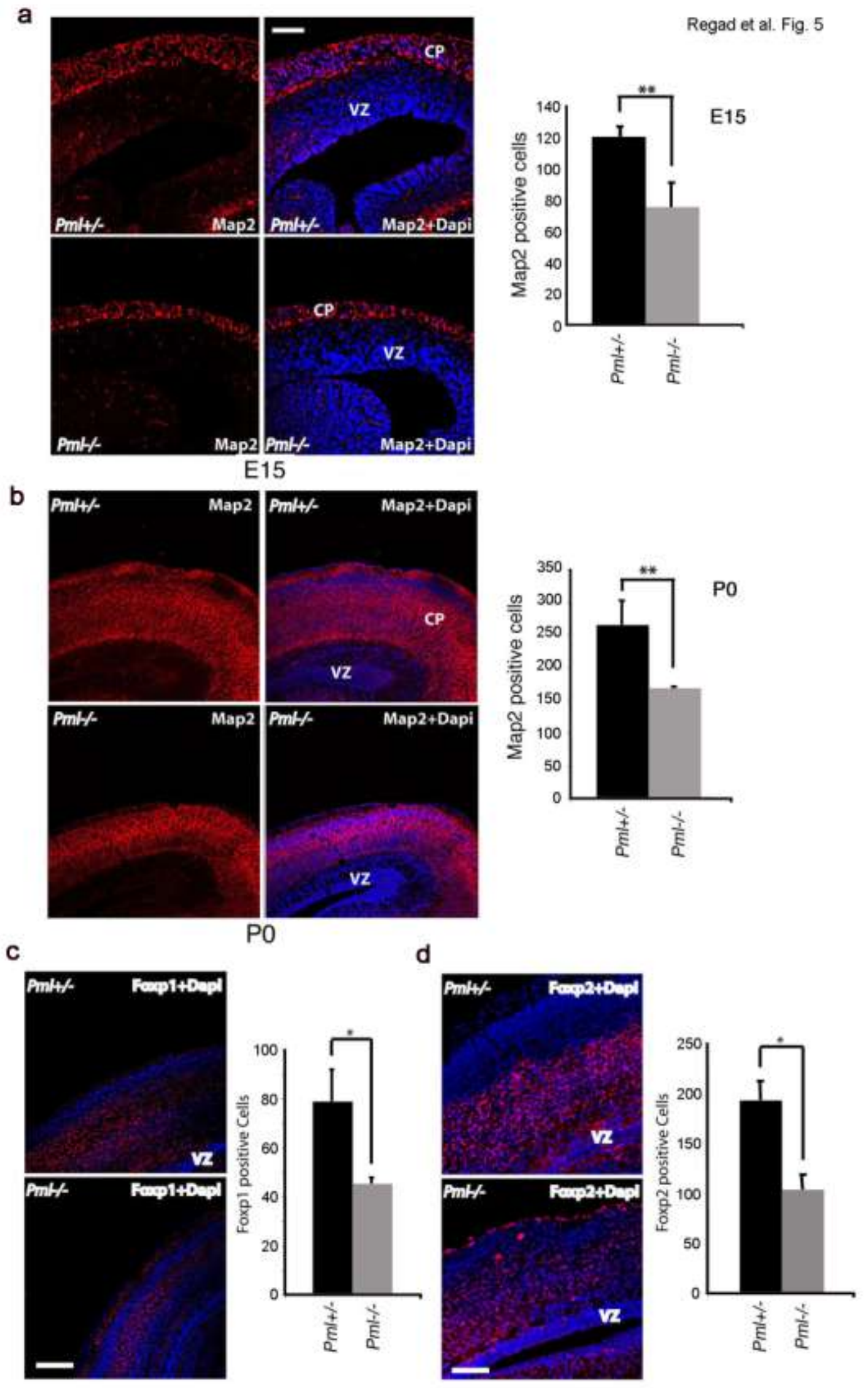


a

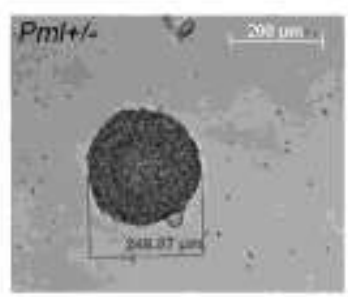

b

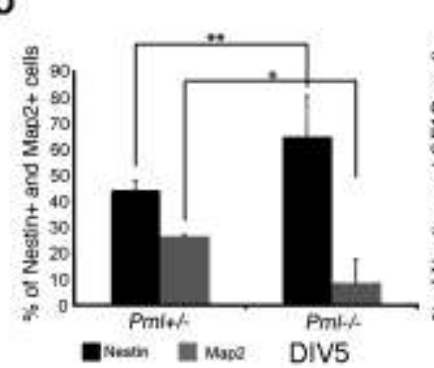

C

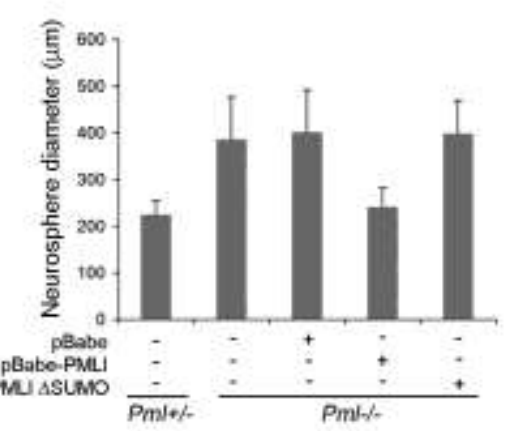

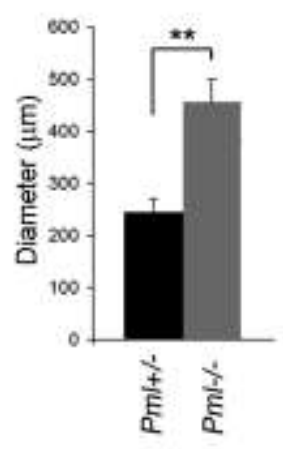
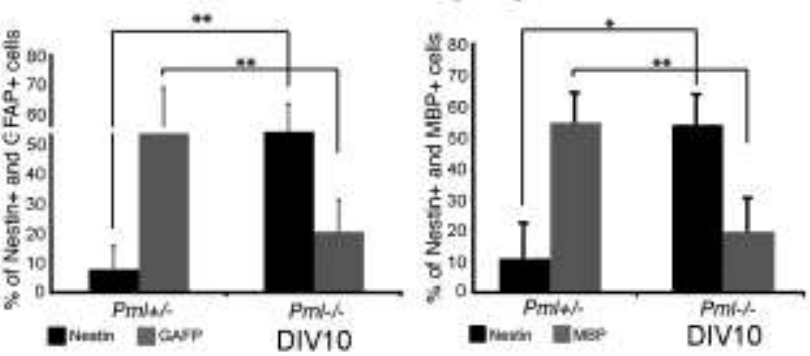

d

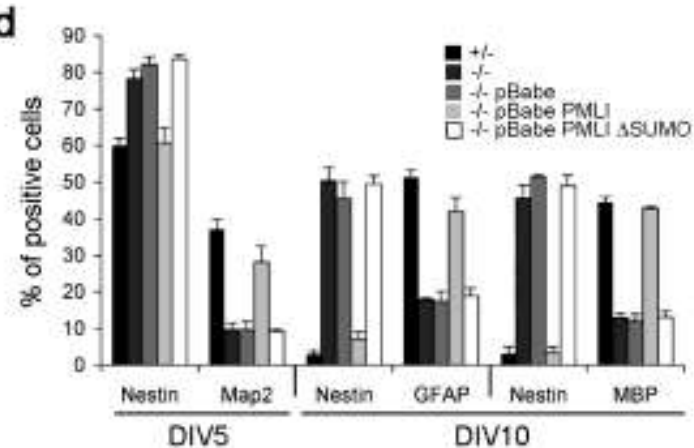




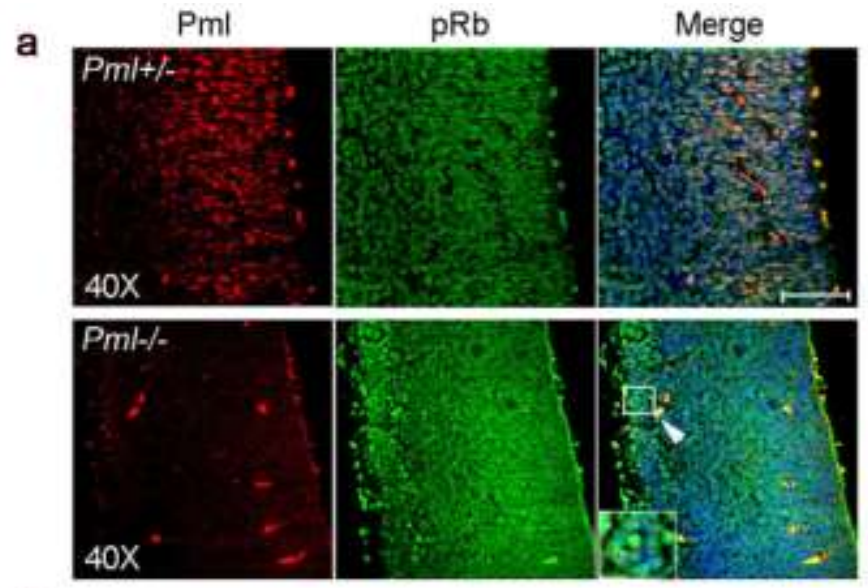

Regad et al. Fig, 7

b

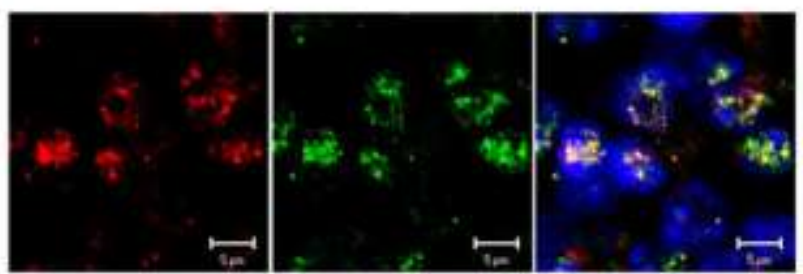

C

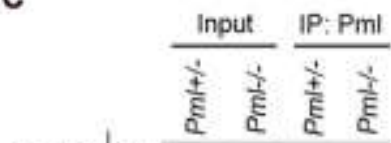

IB: PRB $\mid \begin{aligned} & 1500 \\ & 100- \\ & 1000\end{aligned}$

IB: Pml

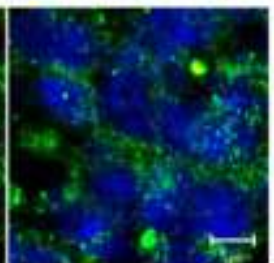




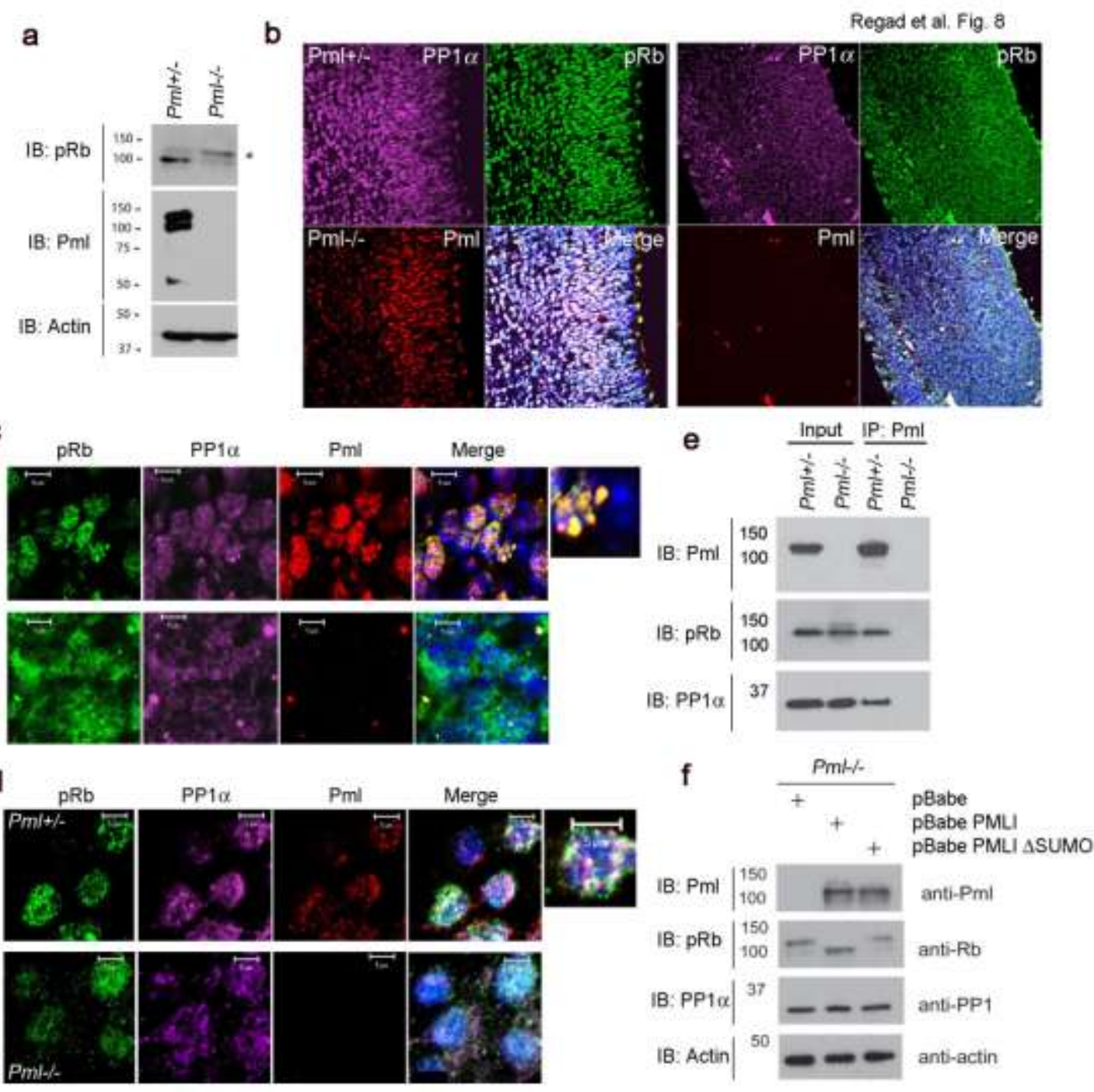

